Western University Scholarship@Western

Chemistry Publications

Chemistry Department

Fall 11-1-2017

\title{
Dendritic Plasmonics for Mid-Infrared Spectroscopy
}

Francois Lagugne-Labarthet

University of Western Ontario, flagugne@uwo.ca

Gregory Q. Wallace

Western University

Hayden C. Foy

Western University

Scott M. Rosendahl

Canada Light Source

Follow this and additional works at: https://ir.lib.uwo.ca/chempub

Part of the Physical Chemistry Commons

Citation of this paper:

Lagugne-Labarthet, Francois; Wallace, Gregory Q.; Foy, Hayden C.; and Rosendahl, Scott M., "Dendritic Plasmonics for Mid-Infrared Spectroscopy" (2017). Chemistry Publications. 100.

https://ir.lib.uwo.ca/chempub/100 


\title{
Dendritic Plasmonics for Mid-Infrared Spectroscopy
}

Gregory Q. Wallace, ${ }^{\dagger}$ Hayden C. Foy,${ }^{\dagger}$ Scott M. Rosendahl, ${ }^{\ddagger}$ and François Lagugné-Labarthet ${ }^{\dagger, *}$

${ }^{\dagger}$ Department of Chemistry and the Centre for Advanced Materials and Biomaterials Research, University of Western Ontario, 1151 Richmond Street, London, Ontario N6A 5B7, Canada

\$Canadian Light Source Inc., 44 Innovation Boulevard, Saskatoon, Saskatchewan S7N 2V3, Canada

\begin{abstract}
Metallic nanostructures that exhibit tailored optical resonances spanning from the near to midinfrared spectral range are of particular interest for spectroscopic and optical measurements in these spectral domains that can benefit from localized surface-enhancement effects. Plasmon resonances shifted in the near or mid-infrared range could be used to further enhance the excitation and/or the emission of an optical process. Surface-enhanced infrared absorption (SEIRA) is one of such processes and can particularly benefit from plasmon-enhanced local fields yielding an increase in sensitivity towards the detection of an analyte. Herein, we have fabricated a series of gold dendritic nanostructures, prepared by electron-beam lithography, that exhibit plasmon resonances spanning the near and mid-infrared spectral regions. We explore the influence of the number of branches of the dendritic structures, as well as the length of each generation together with the overall effect of the shape and symmetry on the resulting optical
\end{abstract}


resonances. The creation of new resonances that appear upon newer fractal generation are explained in light of an hybridization model. Selected structures were then evaluated for SEIRA measurements towards an analyte.

\section{Introduction:}

The design and fabrication of conductive nanostructures for plasmon-enhanced spectroscopy has become a field of intense research due to their application in molecular sensing and biosensing. ${ }^{1-5}$ Under ideal conditions, extreme sensitivity can be reached, pushing the performances of optical measurements in terms of spatial resolution, ${ }^{6-7}$ and sensitivity down to the single molecule level. ${ }^{8-10}$ Metallic nanostructures with rational dimensions and shapes can, in ideal experimental conditions, locally enhance and confine an electromagnetic field that can be used as a local antenna either in collection (enhancement of the impinging light) or in emission (enhancement of the emitted field). ${ }^{11}$ When a molecule of interest is placed in these nanoscale regions of electromagnetic enhancement, the magnitude of the enhanced vibrational spectra depends on numerous factors, including geometrical factors (i.e. the design of the structure with respect to an excitation wavelength and an input polarization), distribution and density of the molecular species over the structure and of the considered optical process. The field of molecular plasmonics relies on this interaction, and has been exploited to a variety of spectroscopic techniques, most notably for surface-enhanced Raman spectroscopy (SERS), tip-enhanced Raman spectroscopy (TERS) and surface-enhanced infrared absorption (SEIRA). ${ }^{12-14}$ In SERS or TERS, since the enhancement factor varies with the fourth power of $E_{/} E_{0}$, a minimal local field enhancement of a 10 fold factor will therefore yield a predicted enhancement of $10^{4} .{ }^{15}$ Many other linear or nonlinear optical measurements can benefit from such enhancement that scales 
with the considered optical process. ${ }^{16-18}$ In multiple waves mixing processes, the first difficulty is to enhance multiple wavelengths on distinct spectral ranges with a given structure. Being coherent processes, the second difficulty in diffraction limited nonlinear waves is to keep phase matching conditions: the nonlinear sources enhanced by the nanostructure must add up in phase to enable frequency conversion. ${ }^{19}$ Last, depending on the considered nonlinear process, this enhancement may depend on the symmetry of the metallic nanostructure. ${ }^{18,}{ }^{20}$ Keeping in mind all these spatio-temporal critical factors, the possibility to tune multiple resonances over a large spectral domain could be further exploited in nonlinear optical vibrational spectroscopy such as sum-frequency generation or coherent anti-stokes Raman processes, yielding higher sensitivity. ${ }^{21}$

Critical to the field of molecular plasmonics is the tailoring of the localized surface plasmon resonance (LSPR) of the nanostructure such that it is in resonance or pre-resonance conditions with the impinging and or the emitted light. This is commonly achieved through a variety of methods including the alteration the chemical nature of the conductive metal, adjusting the size and shape of the nanostructures, the configuration of the nanostructures arrangement or changing the dielectric constant of the media that surrounds the platform. The development of plasmonic structures that exhibit SERS compatibility is relatively straightforward as it is only necessary to have a resonance that covers a narrow spectral region that both overlaps the excitation and the Raman shifted photons. Expanding into the infrared remains a greater challenge as it is necessary to have enhancement over a broader spectral region $\left(500-4000 \mathrm{~cm}^{-1}, 2.5-20 \mu \mathrm{m}\right)$.

To achieve the enhancement in the infrared range, different conceptual approaches can be undertaken. One means of achieving enhancement in the mid-infrared is through the use of colloidal metallic nanoparticles. ${ }^{22-24}$ Although these platforms can be readily prepared, they often suffer from low enhancement and offer poor reproducibility. In addition, the use of a solvent to 
keep the integrity of the colloidal particles can be detrimental to the optical measurement. To overcome these particular limitations, structures prepared by lithographic techniques have emerged as means of fabricating structures that have LSPRs in the infrared. One of the most common classes of structures are nanorods. An advantage of this type of structure is that they can be readily produced using a variety of lithographic techniques including electron-beam lithography (EBL), ${ }^{13,25-28}$ nanostencil lithography, ${ }^{29-30}$ nanoimprint lithography,${ }^{31}$ and direct laser writing lithography. ${ }^{32-33}$ When fabricated as isolated structures, or as dashed lines, a single absorption is most often observed. Although it can be possible to tune the absorptions of such structures to a narrow portion of the infrared spectral region, introducing additional resonances, beyond the multipolar resonances, using a single rod is not possible.

Introducing multiple absorptions in the infrared spectral region can be achieved by incorporating structures with distinct sizes within the probed region. ${ }^{34-35}$ In these studies, the absorption of a given structure is individually tailored to a particular wavelength. By having multiples structures, multiple resonances are introduced. Another alternative to introducing multiple absorptions is to superimpose the nanostructures orthogonally to each other. ${ }^{36}$ The resulting structure is capable of generating different absorptions simply by rotating the polarization of the impinging light. Although these methodologies have been successfully applied to measurements involving SEIRA, the resulting absorptions of the nanostructure are generally too narrow for the mid-infrared range, thus requiring many variations of the structure to yield multiple resonances with optimized spectral overlap. SEIRA has followed the early developments of SERS, ${ }^{37-39}$ but only recent work by Neuman et al. has shed a new light on the mechanism of SEIRA.$^{40}$ Briefly, the role of scattering and absorption by the nanostructured metallic antenna were elegantly modelled highlighting that maximum enhancement was 
observed when both mechanisms, i.e. scattering and absorption, had similar spectral maxima magnitude. From such modelling work on linearly shaped antennas, it was concluded that tailoring the ratio between absorption and scattering losses could yield optimal structures for SEIRA. Such tailoring could be done by tuning the dimension and the aspect ratio of the antenna. ${ }^{40}$

In the context of nanomaterials with resonances in the infrared spectral range and keeping in mind the observations reported above for vibrational spectroscopy applications, fractal and fractal-like structures have emerged as an interesting class of structure that are capable of exhibiting a greater number of resonances. ${ }^{41-48}$ For many of these structures, nanorods and rodlike structures are used as the base units and are repeated radially, such as in the example of the Cayley Tree. ${ }^{42}$ Introduced as a plasmonic fractal, the Cayley Tree structures inscribed on quartz substrates showed spectral resonances that could be finely tuned between 880 and $4500 \mathrm{~nm}$. However, no resonances were observed beyond $4500 \mathrm{~nm}$ due to the use of a quartz substrate that fully absorbs infrared light beyond this wavelength. ${ }^{49}$ Furthermore, it is likely that due to the overall small size of the individual nanorods $(100-180 \mathrm{~nm})$ comprising the Cayley Tree, a significantly high number of generations would have been required to prepare structures with compatibility in the mid-infrared range. Finally, although the optical properties were well explored, no measurements were performed to ascertain the applicability of such a structure to analyte detection by SEIRA. In this study, we further expand on the use of nanorods as a means of generating dendritic fractals. In particular, we explore larger sized nanorods with different number of branches for the starting generation, along with the higher order generations as a means of preparing dendritic fractals that are compatible with SEIRA spectroscopy. 
Here, EBL is used to prepare these structures onto $\mathrm{CaF}_{2}$ optical windows that are mid-infrared compatible. With a resolution between $10-20 \mathrm{~nm},{ }^{50} \mathrm{EBL}$ is ideally suited to the fabrication of dendritic fractals in particular for the smallest structures present on the highest generations. Utilizing synchrotron radiation as the source of infrared light for our measurements, we begin by using a three-branched dendritic fractal to explore how the optical properties of the dendritic fractal are altered as higher-order generations are developed. Through the use of finite difference time domain (FDTD) calculations, we evaluate the absorption spectra of the structure to the fractal composition at the fourth-order generation and we establish the wavelength spatial distribution of the plasmon resonances over the selected structure. We then introduce various means of tailoring the optical properties of the dendritic fractal across the near- and mid-infrared spectral regions. This is achieved by not only altering the size of the individual nanorods, but also by increasing the number of branches in the first-order generation. It was found that increasing the number of branches results can result in the branches becoming too tightly packed. Therefore, we also prepare truncated dendritic fractals as a means of further tuning the optical properties and measured their mid-infrared resonances. Last, the prepared platforms were functionalized with an analyte to demonstrate the compatibility of the structures for molecular plasmonics in the mid-infrared.

\section{Experimental:}

\section{Electron-Beam Lithography}

$\mathrm{CaF}_{2}$ windows (13 mm diameter x $2 \mathrm{~mm}$, Spectral Systems LLC, NY, USA) were first cleaned using reactive $\mathrm{O}_{2}$ plasma for 20 minutes to ensure adhesion of the thin film of resist. The windows were then coated with a $100 \mathrm{~nm}$ thick layer of poly(methyl methacrylate) (PMMA, A2 950 resist (MicroChem Corp., MA, USA)). A thin layer of AquaSave (Mitsubishi Rayon 
America Inc., NY, USA) was subsequently applied above the PMMA layer. A Leo Zeiss 1530 SEM was used to perform all electron beam lithography using a $30 \mathrm{kV}$ EHT acceleration voltage and $10 \mu \mathrm{m}$ aperture. An area exposure dose of $260 \mu \mathrm{C} / \mathrm{cm}^{2}$ was used for the writing of each structure, and each array was fabricated as a patch with an area of $50 \times 50 \mu \mathrm{m}^{2}$. Following exposure, the solutions were developed (water to remove AquaSave, and 1:3 methyl isobutyl ketone: isopropanol (Microchem Corp., MA, USA)), and dried under air. Electron beam evaporation was used to deposit a $3 \mathrm{~nm}$ titanium adhesion layer, and $20 \mathrm{~nm}$ layer of gold at a rate no greater than $0.5 \AA / \mathrm{s}$. The liftoff of remaining PMMA was performed in acetone (CHROMASOLV, Sigma-Aldrich, MO, USA). Scanning electron micrographs of the structures were collected with a Leo Zeiss 1530 SEM. Prior to imaging the structures, the sample was coated with $5 \mathrm{~nm}$ of osmium.

\section{Infrared Absorption and Surface-Enhanced Infrared Absorption}

Fourier transform infrared (FT-IR) spectroscopy measurements were performed at the Mid-IR beamline synchrotron facility located at the Canadian Light Source (Beamline 01B-01). The beamline end station consists of a Bruker Optics Vertex 70v FT-IR Spectrometer coupled to a Hyperion 3000 IR Microscope (Bruker Optics, MA, USA). Light was focused and collected in absorbance mode using a $36 \times$ objective (NA 0.65). The input source coming from the synchrotron beam was linearly polarized. The collected light was measured using a narrowband fast DC coupled mercury cadmium telluride (MCT) (liquid nitrogen cooled) Kolmar (Kolmar Technologies, Inc., MA, USA) detector. Measurements were collected from $8000-800 \mathrm{~cm}^{-1}$ with a spectral resolution of $4 \mathrm{~cm}^{-1}$. Each spectrum is the average of 512 spectra. For surfaceenhanced infrared absorption measurements, the samples were functionalized for 6 hours in a freshly prepared $10^{-3} \mathrm{M}$ solution of 4-nitrothiophenol (4-NTP) (Sigma-Aldrich, MO, USA) 
prepared in dry ethanol. After 6 hours, the solution was dipped in dry ethanol to remove any unbound 4-NTP and was dried under air.

\section{Electromagnetic Field Modelling}

Finite Difference Time Domain (FDTD) modelling (Lumerical) was used to simulate the absorption and electromagnetic fields of the dendritic fractals. Shown in the text, the individual nanorods that the dendritic fractal was comprised of had lengths of $200 \mathrm{~nm}$, widths of $50 \mathrm{~nm}$, heights of $20 \mathrm{~nm}$ for gold, and $3 \mathrm{~nm}$ of titanium placed beneath the gold. Palik dielectric values for gold and titanium were used for the FDTD calculations. ${ }^{51}$ The structures were placed on a substrate with a constant refractive index of 1.42 representing the $\mathrm{CaF}_{2}$ window, and had a thickness of $250 \mathrm{~nm}$. Periodic boundaries on the $x$ and $y$ axes conditions were no smaller than $700 \mathrm{~nm}$, and were representative of the periodicity of the fabricated structure. Last, a perfectly matched layer (PML) was used in the $z$ axis. Mesh sizes of $7.5 \mathrm{~nm}$ were used in the $x$ and $y$ axis and $4 \mathrm{~nm}$ in the $z$ axis. In an attempt to mimic the experimental conditions considering the use of an objective with a numerical aperture of 0.65 (40 degrees half angle), calculations were performed at distinct incidences and showed no major effect on the position of the resonances in the modelled spectra.

\section{Results and Discussion:}

\section{General Optical Properties of Dendritic Fractals}

The dendritic fractals shown in Figure 1 are based on the Cayley Tree structures first used by Halas et al. ${ }^{42}$ In their work, the structures, that were inscribed up to the 3 rd generation, did not show any resonance in the mid-infrared range due to the cut-off wavelength of the quartz substrates $(4.5 \mu \mathrm{m}) .^{49}$ Here, we have made use of $\mathrm{CaF}_{2}$ that has a cut-off closer to $10 \mu \mathrm{m}$, providing access to the fingerprint region from 1000 to $1800 \mathrm{~cm}^{-1} \cdot 33,35$ 

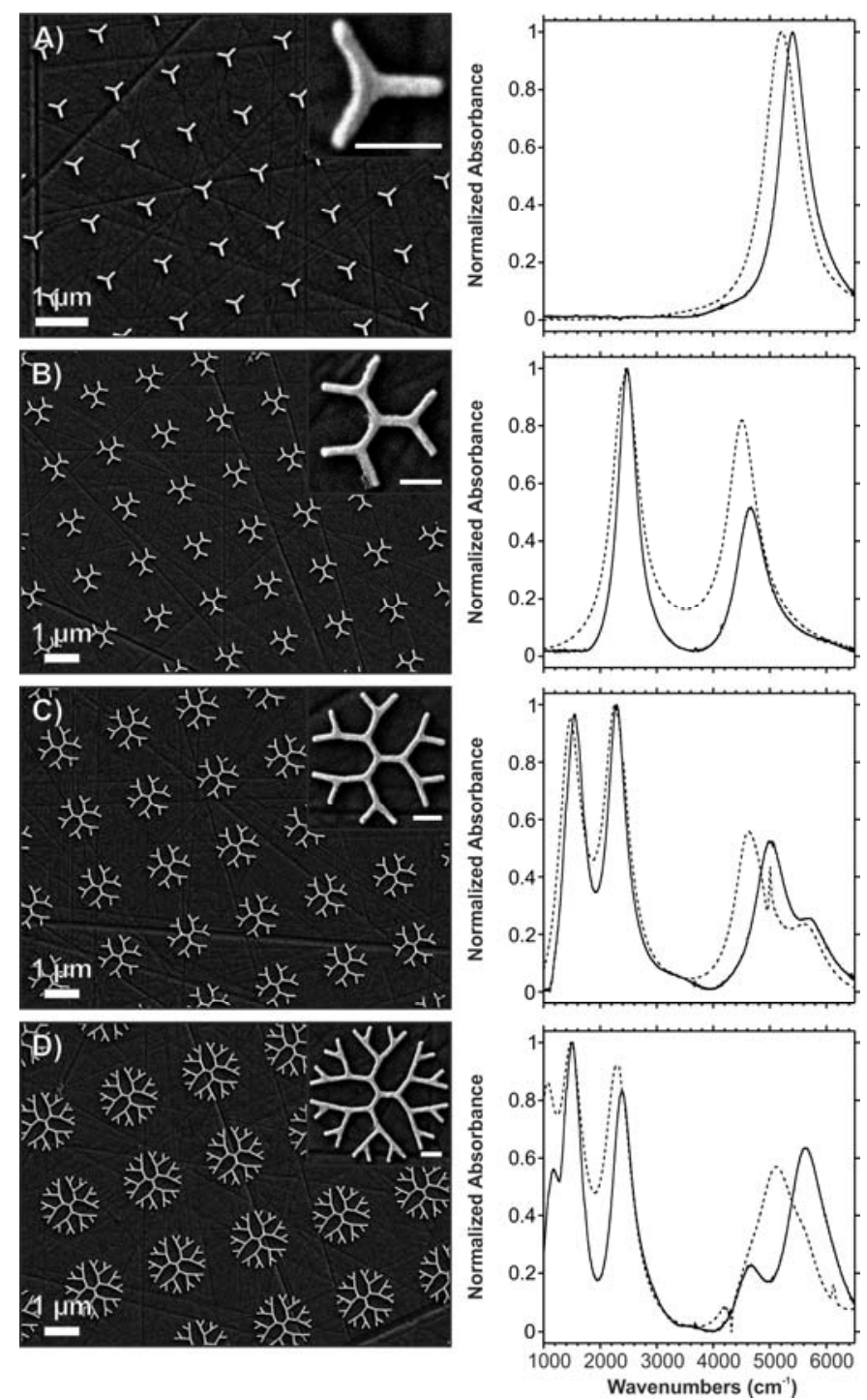

Figure 1. Scanning electron micrograph of dendritic fractals in the A) first, B) second, C) third, and D) fourth-order generations. E-H) Corresponding experimental (solid line) and calculated (dashed line) absorption spectra for each of the generations. The experimental spectra were collected on the same sample as the SEM images. The scale bar in the inset SEM images is 200 nm.

To investigate the influence of fractal generation on the optical properties, structures were fabricated up to the fourth-order generation, as shown in the SEM images Figure 1A-D. Each 
gold rod of the dendritic fractal had a thickness of $20 \mathrm{~nm}$, a width of $50 \mathrm{~nm}$, and the rod elements forming the fractals shown in Figure 1A-D had lengths of $200 \mathrm{~nm}$. Beneath each structure was a titanium adhesion layer of $3 \mathrm{~nm}$. The structures were fabricated in $(50 \times 50) \mu \mathrm{m}^{2}$ arrays, with varying periodicities. A sufficiently large gap (at least $700 \mathrm{~nm}$ ) between the adjacent fractals was used to ensure that no plasmon coupling occurred between fractals. Within the patch of structures, it was found that the structures were fabricated with a high degree of reproducibility (Figure S1).

The infrared absorption measurements taken for each sample shown in Figure A-D are shown in Figure 1E-H together with the calculated spectra. In order to maximize the signal-to-noise ratio of our infrared measurements, the mid-infrared beamline of a synchrotron was used. In a previous study done by our group, a comparison between the use of the CLS mid-infrared beamline synchrotron source and a conventional FT-IR source showed no change in the spectral position for the absorptions of superimposed nanoprisms. ${ }^{35}$ Thus, the spectral location of the absorptions can be described as being source independent.

Beginning with the first-order generation, a single absorption at $5500 \mathrm{~cm}^{-1}$ was observed. Although such an absorption is not particularly relevant for SEIRA, it may be of value for other surface-enhanced spectroscopies, specifically surface-enhanced near-infrared absorption (SENIRA), ${ }^{52}$ and surface-enhanced Raman spectroscopy (SERS). ${ }^{53}$ As a technique, SENIRA probes the vibrational overtones associated with the infrared vibrational modes that can be probed by SEIRA. ${ }^{52}$ Although not explored in this study, the ability to have a plasmonic platform that is compatible with both SEIRA and SENIRA may be of interest, as it could provide a more complete vibrational fingerprint for a molecule of interest. SERS measurements predominantly rely on the use of visible light. More recently, there has been an interest in developing platforms 
compatible with longer wavelengths, such as $1550 \mathrm{~nm}\left(6452 \mathrm{~cm}^{-1}\right)$ because these longer wavelengths are retina-safe. ${ }^{53}$ Although the results of the dendritic fractal (Figure 1E-H) do not have absorptions at such a wavelength, it should be possible to tune the absorptions to such a wavelength. In doing so, the dendritic fractal could then potentially be used for SEIRA, SENIRA, and SERS.

For the dendritic fractals, we observed that in the $\mathrm{N}^{\text {th }}$ generation, the infrared spectra were dominated by $\mathrm{N}$ resonances. As higher-order generations were probed $(\mathrm{N}>1)$, additional resonances were found at lower wavenumbers. As it has been previously described, the dominant absorptions presumably correspond to various dipolar modes of the structure at each generation. Reciprocally, the weaker absorptions near $5800 \mathrm{~cm}^{-1}$ and $4700 \mathrm{~cm}^{-1}$, were observed for the thirdorder generation (Figure 1G) and fourth-order generation (Figure 1H) respectively, and are assigned to the quadrupolar resonances of the structure. The dendritic fractal was also inscribed for the fifth-order generation, shown in Figure S2A. However, we were unable to introduce any new dominant absorption bands at lower wavenumbers (Figure S2B). This is most probably because in the fourth-order generation, the lowest energy absorption was near $1200 \mathrm{~cm}^{-1}$, very close to the cut-off limitation of the $\mathrm{CaF}_{2}$ substrate. At the fifth-order generation, the new low energy absorption would likely be lower than $1000 \mathrm{~cm}^{-1}$, and could not be probed. So although higher-order generations are within the fabrication limitations, they cannot be exploited in the mid-infrared range.

In order to correlate the resonances and their spatial localization with the generation order of the dendritic fractal, a plasmon hybridization model was used. ${ }^{54-56}$ In Figure 2, the calculated spectra and associated field enhancements for the first four generations of the dendritic fractals are calculated between 1000 and $7000 \mathrm{~cm}^{-1}$ using finite difference time domain (FDTD) 
calculations. When going from the first-order generation to the fourth-order generation, the number of resonances increases from 1 to 5 . Since these structures are composed of concentric features (i.e. the dendron that forms the iterative fractal components), the hybridization model appears relevant to explain the major resonances together with the electric field distribution. For the lower-order generations of the fractal, the initial resonance(s) splits into two resonances with high (HE) and low (LE) energies (Figure 2). When going from the first to the second-order generations, the initial single mode that appears at $\lambda=1.92 \mu \mathrm{m}$ is split into two modes with wavelengths of $\lambda=2.22 \mu \mathrm{m}$ (HE) and $\lambda=4.06 \mu \mathrm{m}$ (LE). To better understand this splitting, we proposed a hybridization model that combines the structure from the first-order generation (G1), along with the outer-most structures that were introduced in the second-order generation (G2G1) (Figure S3). This approach provides the most physically acceptable energetic assignments accounting for the modes of G1 and G2-G1.

The third-order generation shows four modes hybridized from the second-order generation structure located at $\lambda=1.75 \mu \mathrm{m}$ (HE1) and $\lambda=6.62 \mu \mathrm{m}$ (LE1) as well as $\lambda=2.14 \mu \mathrm{m}$ (HE2) and $\lambda=4.42 \mu \mathrm{m}$ (LE2), respectively (Figure 2). A similar approach was used for the hybridization model (Figure S3), where the parent structures of the second-order generation $(\mathrm{G} 2)$ and the outer-most structures of the third-order generation (G3-G2) were combined. Moving to the fourth-order generation shows a more complex spectrum with overlapping resonances. Here, the electric field enhancement is calculated only for the five major resonances derived from the previous generation. Once again, the hybridization of the plasmon modes between the previous generation and that of the newly introduced structures can tentatively be used to explain the newer resonances that appear at $\lambda=1.78,1.95,4.38,6.72$ and $9.36 \mu \mathrm{m}$. When higher order 
generations above the fourth-order are calculated, very little spectral difference can be observed experimentally (Figure S2A) or from calculation (not shown).

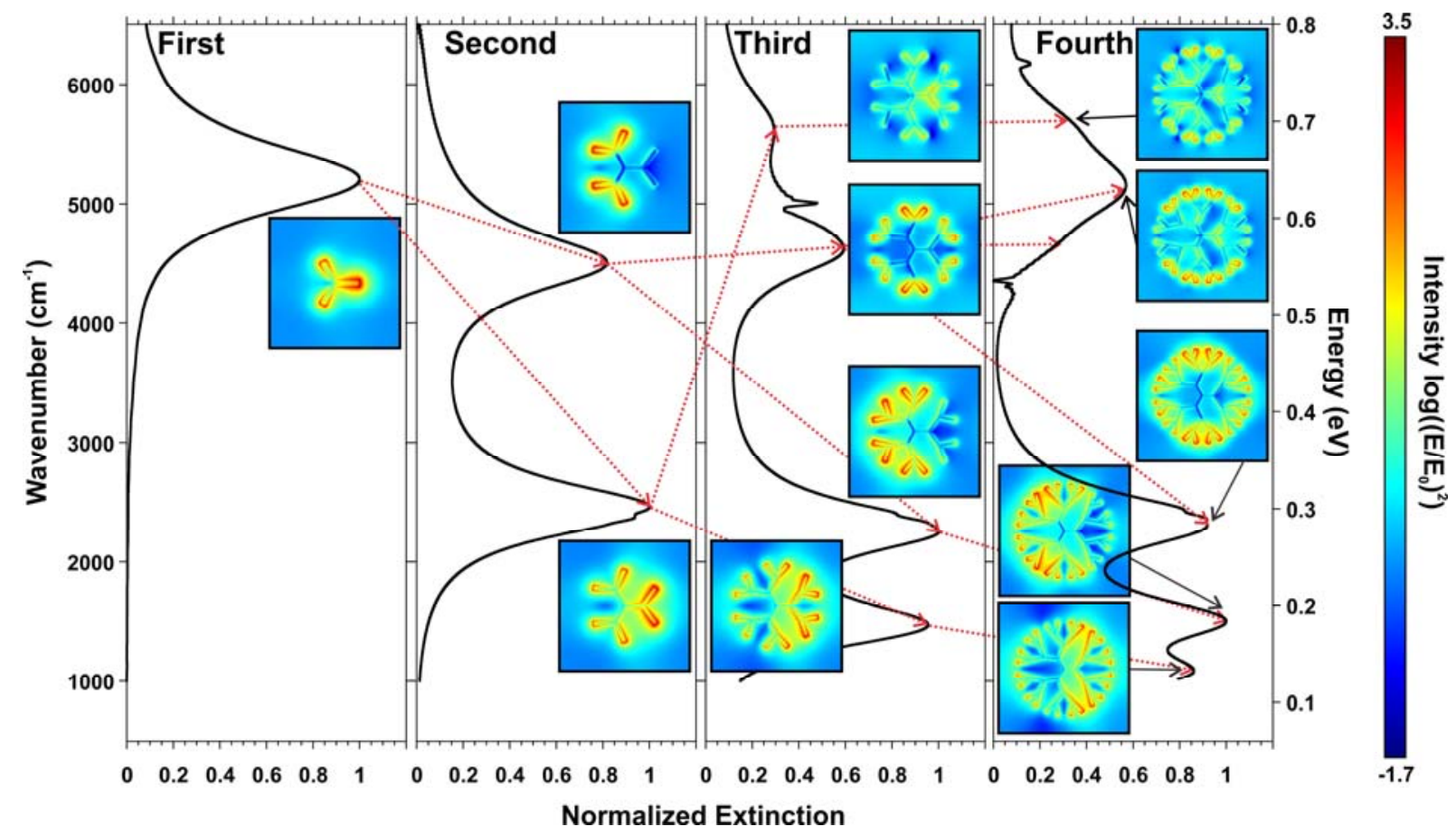

Figure 2. Normalized extinction spectra for first, second, third, and fourth-order generation dendritic structures with individual rod lengths of $200 \mathrm{~nm}$. For each generation, the normalized electric field distribution $\left(\mathrm{E} / \mathrm{E}_{0}\right)^{2}$ under horizontal polarization was calculated for each resonance and displayed using log scale for clarity. The plasmon hybridization (red dashed line) is shown upon iteration of the fractal generation. The wavelengths associated with the multiple resonances in the third-order generations are $\lambda=1.75,2.14,4.42,6.62 \mu \mathrm{m}$, and $\lambda=1.78,1.95,4.38,6.72,9.36$ $\mu \mathrm{m}$ for the fourth-order generation.

Details on the resonances observed in the fourth-order generations structures are provided in Figure 3. The electric field distribution over the structure were calculated for selected input wavelengths that correspond to the four major resonances as shown in the spectrum of Figure $1 \mathrm{H}$ and Figure 2. Two orthogonal polarizations $\left(0^{\circ}\right.$ and $\left.90^{\circ}\right)$ were selected for these calculations. 

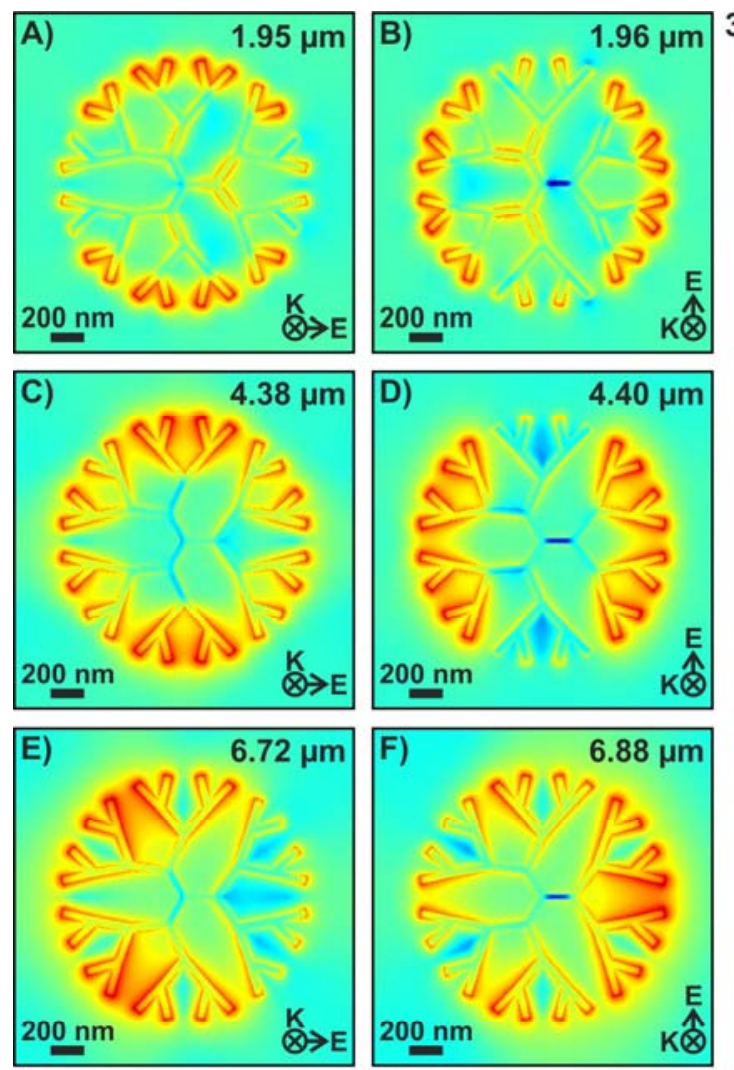

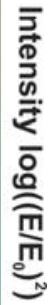
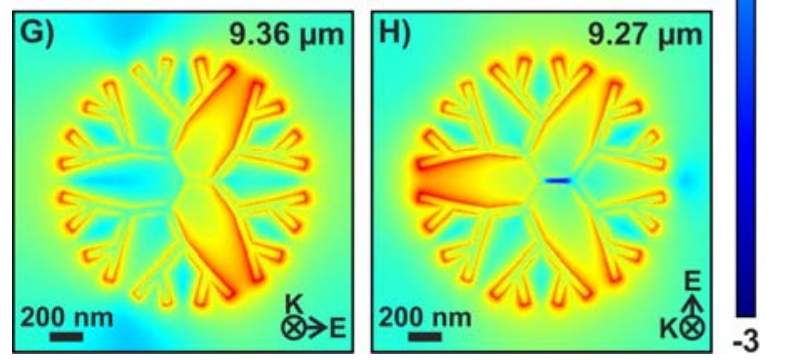

Figure 3. FDTD calculations of the transverse components of the electric field $\left(\left|\mathrm{E} / \mathrm{E}_{0}\right|\right)^{2}: \log$ scale representation at wavelengths corresponding to the absorptions of the fourth-order generation dendritic fractal comprised of gold nanorods with side lengths of $200 \mathrm{~nm}$.

Examining the electric field distribution maps, shown in Figure 3, highlights a well-defined relationship between the fractal order and the spatial distribution. Starting with the highest energy absorptions (Figure 3A,B), the electric field is enhanced in the branches introduced in the fourth generation. By altering the polarization of the impinging light, it is possible to selectively excite the LSPRs across the entirety of the outer periphery of the structure. Moving to the second 
highest energy (Figure 3C,D), the enhancement now incorporates the branches from the third and fourth generations. As the absorptions move to lower energies (Figure 3E-H), each absorption incorporates the branches from an additional generation, until as shown in Figures 3G,H, the LSPR is spread over the whole structure. It is necessary to note that in this work, the absorptions closer to the fingerprint region are the most important since they will be used to enhance the absorption fingerprint of the analyte. Therefore, ensuring that absorption(s) of the structure are in this region is critical. Furthermore, the intensity of the electric field at these regions must also be considered. The electric field distribution map of Figure 3 provides an estimated $\left(E^{2} / E_{0}^{2}\right)$ enhancement of $10^{3.5}$. This enhancement is lies within the range of $10^{2}-10^{5}$ that is experimentally observed for SEIRA compatible nanostructures.

To better visualize the spatial distribution of the multiple resonances, the iso-wavelength maps at the orthogonal polarizations were calculated (Figure 4) ${ }^{57}$ In this representation, each map is first calculated at 44 discrete wavelengths over a spectral range of $1.4-10 \mu \mathrm{m}$. For each polarization, this forms a $3^{\text {rd }}$ rank tensor of dimensions $\mathrm{X}(308), \mathrm{Y}(308)$ and $\lambda(44)$. For each $(x, y)$ spatial location, the tensor is then analyzed along the $\lambda$ direction, and the $\lambda$ max is extracted and assigned to an $(x, y)$ spatial positon. This representation forms a new matrix of $(\mathrm{X}, \mathrm{Y})$ that represents the distribution of the $\lambda_{\max }$ associated with the multiple resonances and that we refer to as the iso-wavelength maps.

These iso-wavelength maps are shown for both orthogonally-polarized input sources (Figure 4B and C). Using the SEM image in Figure 4A for reference, the variation of the wavelength corresponding to the electric field maximum can be seen as an overlap of the results shown in Figure 3. Under varying polarizations of the input electric field, it is once again observed that the outer branches correspond to higher energy wavelengths, whereas the inner most branches 
coupled with the outer branches exhibit stronger electric fields at lower energies. Owing to the configuration of the three-branched dendritic fractal, only a few branches of the structure contribute to the enhancement of the electric field. As such, the fabrication of dendritic fractals with a greater number of initial branches may lead to improved enhancement of the electric field, yielding greater enhancement for measurements in molecular plasmonics. Importantly, the isowavelength maps show an interesting polarization-dependence that can be further exploited. In Figure 4B, the distribution of maximum wavelength is asymmetric with respect to the polarization direction. This implies that the left part of the structure is predominantly subject to shorter wavelength resonances meanwhile the right part display resonances at longer wavelengths. For the orthogonal polarization direction, that does not match any symmetry axes of the structure, the iso-wavelength map shows that longer wavelengths are confined in the inner cavity formed by the longer branches of the structure. Such symmetry effects could potentially be used for optical processes where symmetry of the structure is critical with respect to the input field. The proposed structure could potentially be active for second-order non-linear optical processes due to its absence of an inversion center. This also implies that the rotation of the input polarization in this structure with 3-fold symmetry will enable each plasmon resonance to be tuned on demand in selected part(s) of the structure.
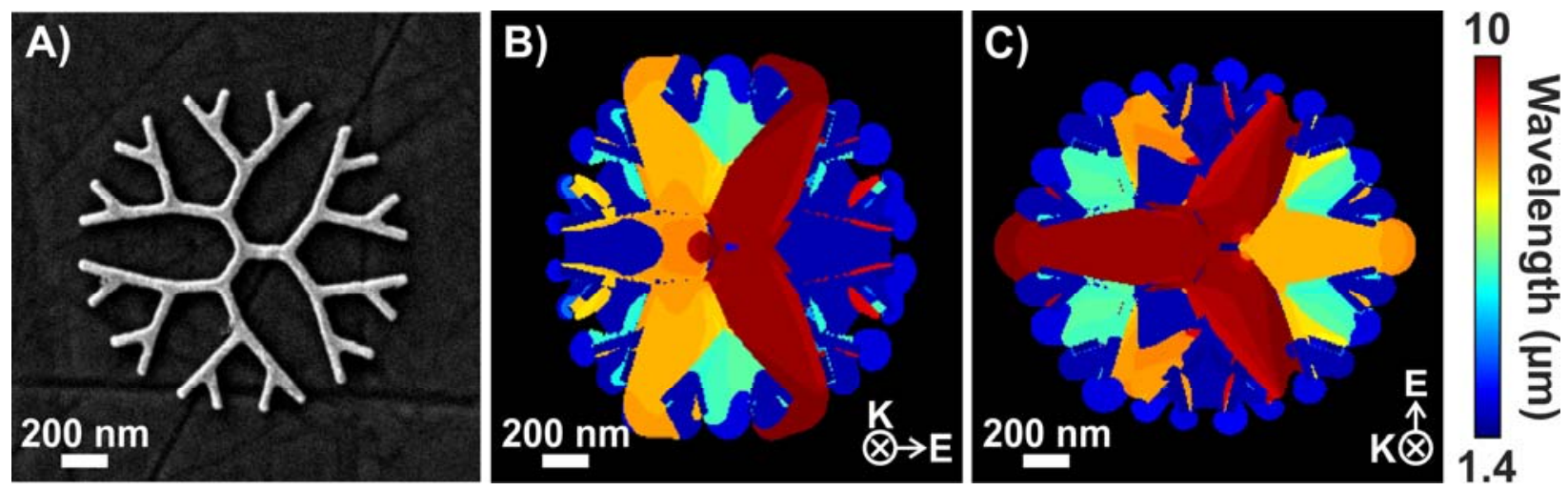

Figure 4. A) SEM image of the fourth-order generation fractal where the side lengths are 200 
$\mathrm{nm}$. Colour coded map of iso-wavelength distribution representing the distribution of the maximum electric field for a B) $x$ - and C) $y$-polarized input field.

\section{Increasing the Size of the Dendritic Fractal}

As has been described, the spatial location of the absorptions is related to the overall shape of the dendritic fractal and the input polarization direction. However, tuning the absorptions of the structure based exclusively on the number of generations is not ideal as it may not be possible to tune absorptions across spectral range spanning from near to mid-infrared. Therefore, to bridge the spectral gap, it is necessary to turn to alternative means for spectral tuning.

A common procedure for tuning plasmonic properties is by altering the size of the individual building blocks that compose the nanostructure. ${ }^{58}$ In this study, the lengths of the individual rods of the dendritic fractal were varied from $200-400 \mathrm{~nm}$. This range of size was selected as it was believed that such structures would offer the ability to have a greater number of absorptions closer to the fingerprint region, as opposed to the sizes previously studied for the Cayley Tree fractal $(100-180 \mathrm{~nm}){ }^{42}$
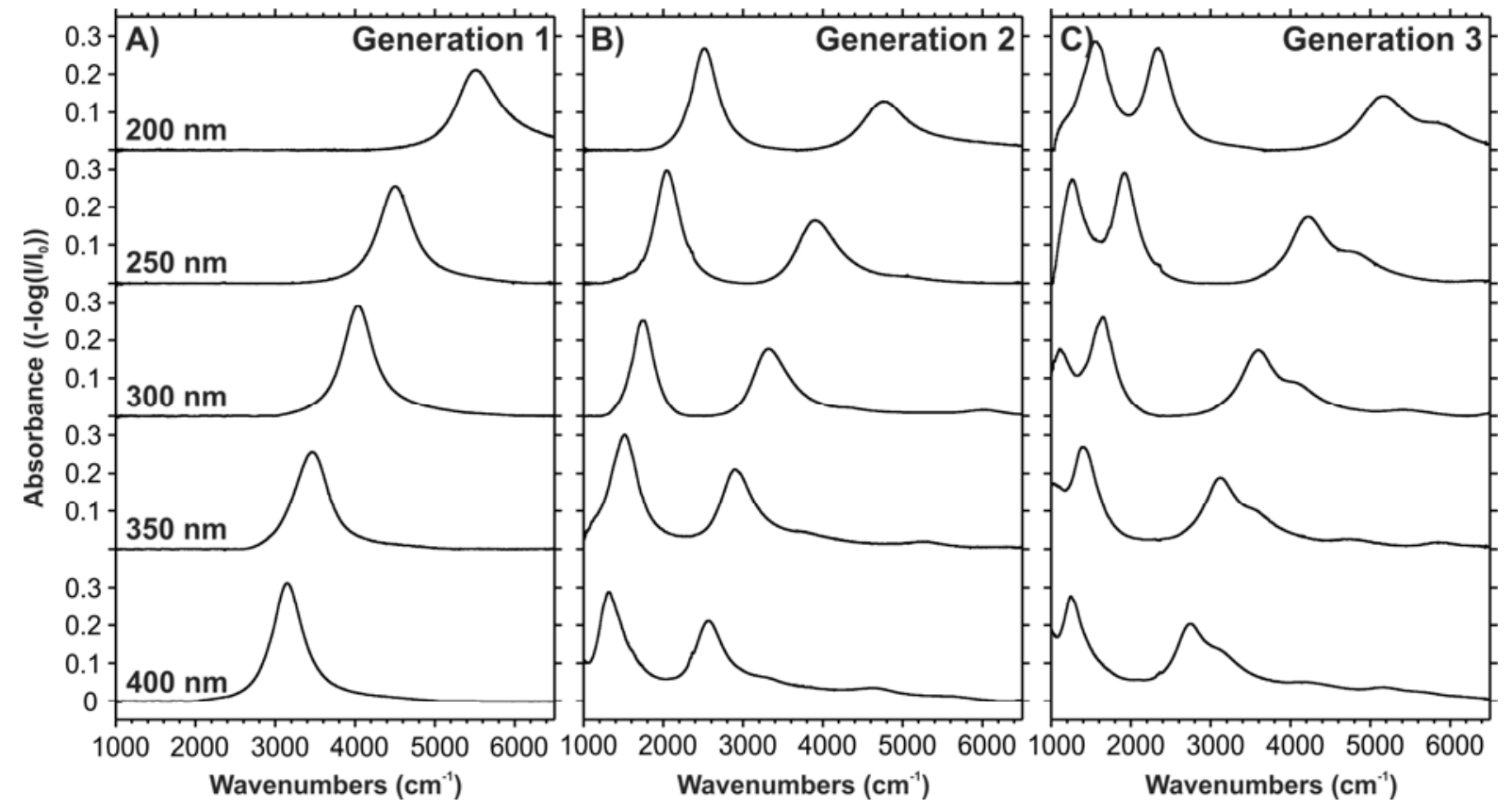
Figure 5. Infrared absorption of three-branched dendritic fractal with different sized nanorods at the A) first-order, B) second-order, and C) third-order generations. The lengths of the rods are indicated in A).

As expected, increasing the size of the individual nanorods within the dendritic fractal results in a red-shift of the absorptions (Figure 5). It was found that for the structures probed, a spectral shift of 6-7 nm was introduced for every $1 \mathrm{~nm}$ increase in the length of the nanorods. With such a high degree of spectral tunability, it was possible to shift the absorption of the first-order generation absorption from $5500 \mathrm{~cm}^{-1}$ to $3150 \mathrm{~cm}^{-1}$ simply by increasing the size of the nanorods from 200 to $400 \mathrm{~nm}$ (Figure 5A). It is important to note that in studies involving isolated nanorods for applications involving SEIRA, the lengths of the rods are required to be longer. For example, gold nanorods with lengths of $710 \mathrm{~nm}$, widths of $60 \mathrm{~nm}$, heights of $55 \mathrm{~nm}$, with a spacing of $50 \mathrm{~nm}$ between adjacent nanorods, had an absorption at $3093 \mathrm{~cm}^{-1}$. ${ }^{59}$ With the dendritic fractals, it is possible to achieve a similar absorption using nanorods that are approximately half the size. This is because in the dendritic fractal, the nanorods are connected to each other. As such, although the individual nanorods are $400 \mathrm{~nm}$, the entire length of the resulting first-order dendritic fractal is $600 \mathrm{~nm}$ along the $x$-, and $692 \mathrm{~nm}$ along the $y$-directions.

Examining the second-order generation dendritic fractal (Figure 5B), the same red-shift of 6-7 $\mathrm{nm}$ spectral shift for every $1 \mathrm{~nm}$ increase in length, is observed for each absorption. The same spectral shift is also observed for the third-order generation in Figure 4C. However, it is necessary to note that increasing the size of the nanorods too much eventually leads to a diminishing return. As was the case for expanding beyond the fourth-order generation, if the individual nanorods become too large (such as 350 and $400 \mathrm{~nm}$ ), it is no longer possible to observe all the absorptions. The absorption corresponding to the global plasmon resonance of the 
structure is lost due to the $\mathrm{CaF}_{2}$ cut-off. Therefore, in addition to the limitation of the number of generations, there is a limitation in terms of structure size that must also be considered when fabricating dendritic structures for applications in plasmonics.

Further tuning of the spectral positions of the absorption could be investigated in future work. For example, when the lengths of the nanorods were increased, this was applied to all rods within the structure. One can envision a structure where the branches corresponding to each generation are of different lengths. The likely result of such a structure is that the total number of absorptions would remain the same whilst a spectral shift is observed for each absorption. If the desire is to have the greatest number of absorptions, an alternative method would be required. Here, each arm of the dendritic fractal would have nanorods with different lengths. The resulting absorption spectrum of such a structure could be viewed as a combination of the spectra obtained when each individual length was studied, as was done in this study (Figure 4).

\section{Increasing the Number of Branches}

Thus far, the emphasis of spectral tuning has been placed on the generation order and the size of the individual nanorods within the structure. Since the shape of the dendritic fractal can be altered by increasing the number of branches within the first generation, there may exist yet another means of tuning the absorptions. Figure $6 \mathrm{~A}$ shows that as the number of branches in the first-order generation increases from 3 to 8 , a noticeable blue shift from $4428 \mathrm{~cm}^{-1}$ to $4815 \mathrm{~cm}^{-1}$ is observed. For the second-order generation fractals, the number of branches is still based on $n-$ 1. This implies that the second-order generation for the dendritic fractal would only be explored for up to $n=6$. This is because as $n$ increases, the available space decreases for the outer generations. At $n=8$, there is likely to be insufficient space to have 7 branches that are fully resolved. The second-order generation fractals exhibit a significant blue-shift for the higher 
energy absorptions (Figure 6B). The lower energy absorption, corresponding the global plasmon resonance is less influenced by the increase in the number of branches in the first generation. Beyond introducing a blue-shift, increasing the number of branches has an additional benefit to the plasmon tuning ability of the structure. As shown in Figure 6A, increasing the number of branches from 3 to 8 decreases the polarization dependence for both orthogonal polarizations. This can be explained based on the overall size of the structure. As the number of branches increases, the resulting dendritic fractal adopts a structure that bears a stronger resemblance to a circle. Since structures such as plasmonic nanodiscs do not exhibit a strong dependence on the input polarization, it is unsurprising that the dendritic fractals with a greater number of branches would exhibit similar optical properties. 

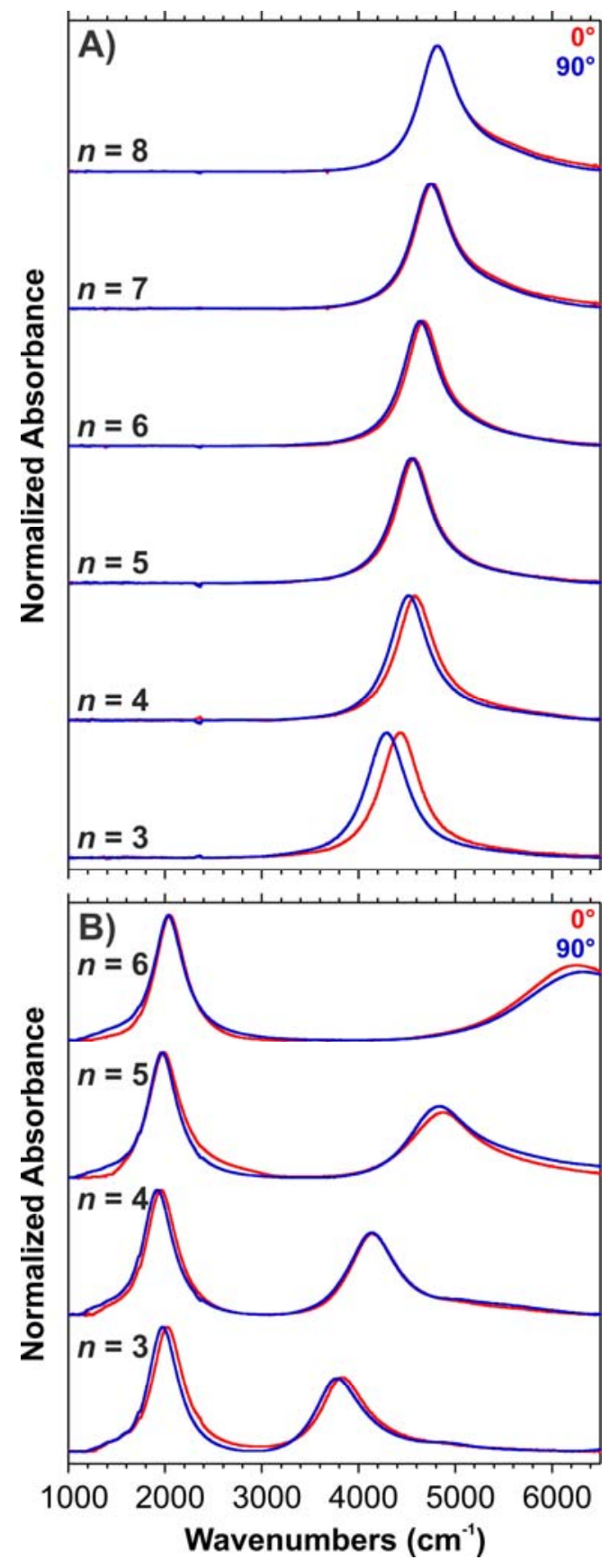

Figure 6. Infrared absorption spectra of dendritic fractals with varying number of inner branches probed under varying polarizations of the input light for the A) first-order and B) second-order generations. The number beside each spectrum corresponds to the number of branches in the first-order generation. The length of the individual nanorods for the resulting structures is 300 nm. 

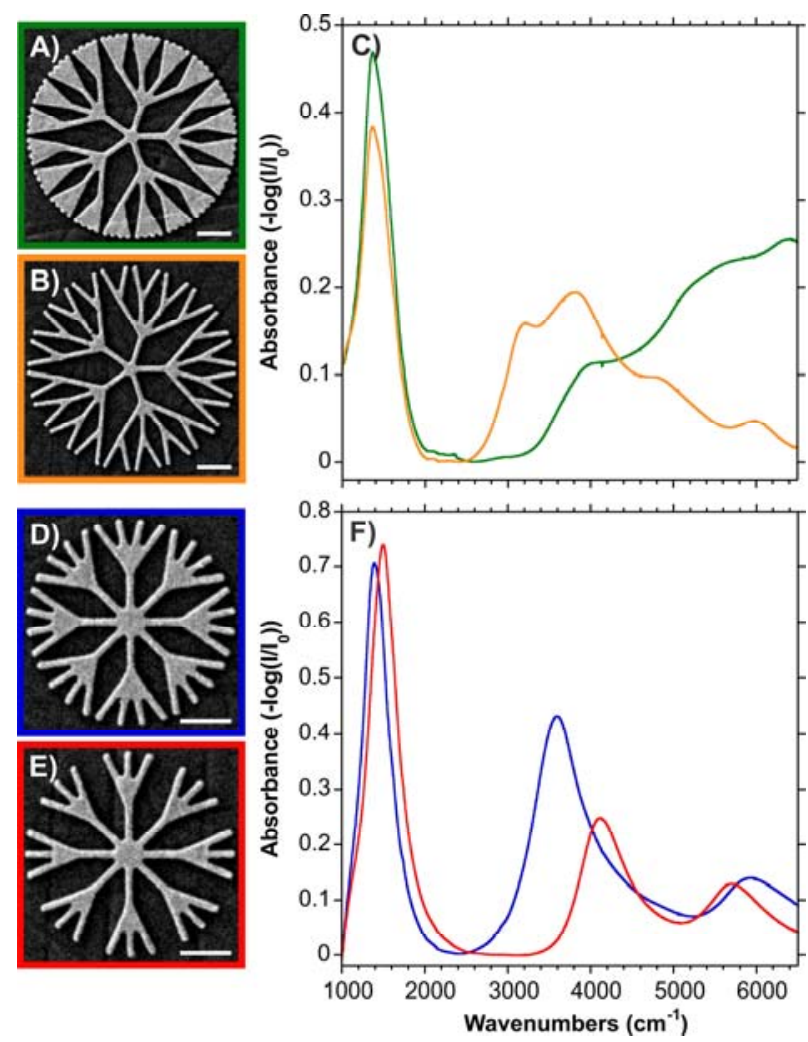

Figure 7. SEM images of the third-order generation of a 5 branched dendritic fractal that is A) original fractal and B) truncated. C) Absorption spectra of the dendritic and truncated fractals from A and B. D and E) SEM images of truncated second-order generation of an 8 branched dendritic fractal. F) Absorption spectra of the truncated fractals from D and E. All of the fractals are comprised of nanorods with lengths of $400 \mathrm{~nm}$. The outline colour of the SEM images corresponds to the colour of the absorption spectrum. The scale bar in the SEM images is 400 nm.

It was possible to fabricate the typical second-order generation dendritic fractal comprised of 5 inner branches (Figure 6B). When fabricated, increasing to the third-order generation results in a dendritic fractal such as the one that is shown in Figure 7A. The branches of the outer generation are not fully resolved, due to a limitation during the fabrication process. As the pattern is being written, rods that are overlapping or are very close together, are exposed to the electron beam 
multiple times. This yields an effective dose that is greater than the nominal exposure dose. Instead of having isolated branches, the branches are instead connected, yielding a "duck foot" like structure. Since it is not possible to add additional space to the pattern, the only means to solve this issue is to remove branches in the outer generation. These new dendritic patterns are hereby described as being truncated dendritic fractals.

Truncating the outer generation of the third-order generation dendritic fractal yields the structure in Figure 7B. A comparison of the absorption spectra of the original and truncated from Figure 7A,B is shown in Figure C. Examining the spectra shows that truncating the fractal does not alter the spectral position of the lower energy absorption. The higher energy absorptions are significantly different between the two spectra. For the original fractal, the higher energy absorptions do not bear a resemblance to the spectral pattern from the second-order generation absorption spectrum (Figure S4A). By truncating the fractal, we not only have our nanorod structure, we also have higher energy absorptions that are closer to those that were observed in the second-order generation dendritic fractal.

The process of truncating the dendritic fractal was also performed on second-order generation structures. This was necessary when the number of inner branches was high. Figures 7D,E show two possible configurations for truncated 8 branched second-order generation dendritic fractals. In the first configuration, three of the outer seven branches are removed (Figure 7D), and in the second four of the branches are removed (Figure 7E). The comparison of the absorption spectra of the resulting structures once again shows that the lower energy absorption, here corresponding to the global plasmon resonance of the structure, is only marginally altered (Figure 7F). Much like the case for the 5 branched structures, the absorptions at higher wavenumbers are altered, most notably the absorptions near 3600 and $4200 \mathrm{~cm}^{-1}$. It is important to note that although there 
is a spectral location difference between these absorptions relative the first order generation, the overall profile of the absorption remains similar (Figure S4B). Much like the case of the third order truncated dendritic fractal from Figure 5B, the truncation prevents the formation of duck feet, allowing for an absorption spectrum that bears a stronger resemblance to the previous generation. When structures are brought in closer proximity to each other, there is a red-shift in the LSPR. In the case of the truncated fractal shown in Figure 7D, the outer branches of each arm are close together $(<50 \mathrm{~nm})$. As such, those branches can couple together, and would yield a redshift in the LSPR relative to a structure that has the rods placed further apart (Figure 7E). For both sets of structures, there are more resonances observed than what would be expected based on the prior results (Figure 1). This is attributed to the increase in size of the nanorods from 200 to $400 \mathrm{~nm}$, which doubles the overall size of the fractal. Due to the overall size of the resulting structures $(2.4 \mu \mathrm{m}$ for Figure 6A,B and $1.6 \mu \mathrm{m}$ for Figure 7D,E), we attribute these other absorptions to the multipolar resonances of the fractal.

\section{Surface-Enhanced Infrared Absorption}

SEIRA measurements were performed on samples functionalized with 4-nitrothiophenol (4NTP). 4-NTP was selected as a model analyte due to the strong absorption of the $\mathrm{NO}_{2}$ symmetric stretching mode at $1340 \mathrm{~cm}^{-1}$ which matches one of the dominant plasmon resonance seen throughout our various dendritic structures. ${ }^{35}$ In experimental conditions where the plasmonic resonance is tuned with the vibrational oscillation of the molecule, a sharp negative dip appears at the molecular vibration frequency of $v_{\mathrm{s}}\left(\mathrm{NO}_{2}\right)$ at $1340 \mathrm{~cm}^{-1}$ as seen in the inset of Figure 8A. ${ }^{60-}$

${ }^{61}$ Such coupling is referred to as a Fano resonance, and is the result of interference between the background of the plasmonic excitation mode and the vibrationally induced molecular dipole governed by the optical near-field confined in the vicinity of the structure. ${ }^{62}$ An anti-resonance 
(sharp dip) is generally observed for weak coupling, as in the case of organic molecules. The observed magnitude of the anti-resonance in the resulting extinction spectra depends on the individual contributions of both absorption and scattering processes, which are mostly dependent on the parameters of the structure. ${ }^{40}$ In order to more easily identify the absorption of the analyte near $1340 \mathrm{~cm}^{-1}$, a polynomial fit was applied to the SEIRA spectra. The resulting fit corresponding to absorption of the structure was then subtracted from the SEIRA spectrum. This was applied to multiple patterns under resonance conditions (i.e. the plasmon resonance is tuned with the molecular vibration) as shown in Figure $8 \mathrm{~B}$. The patterns used in the acquisition of the SEIRA spectra all had resonances between 1230 and $1410 \mathrm{~cm}^{-1}$. The previous work of Vogt et al. demonstrated that a slight red-shift of the vibrational frequency of the analyte relative to the frequency of the plasmon resonance yields the greatest SEIRA enhancement. ${ }^{63}$ The results of Figure $8 \mathrm{~B}$ shows that the strongest signal for the $v_{\mathrm{s}}\left(\mathrm{NO}_{2}\right)$ occurs when the ratio of $\omega_{\mathrm{vib}} / \omega_{\mathrm{res}}=$ 0.96 , consistent with the previously mentioned study.$^{63}$ To illustrate that the signal obtained is the result of the dendritic fractal, a comparison is made between the results of the three branched fourth-order generation $\left(\omega_{\text {vib }} / \omega_{\text {res }}=1.03\right)$ and a $50 \times 50 \mu \mathrm{m}^{2}$ patch of flat gold (Figure S5). No significant signal for the $v_{\mathrm{s}}\left(\mathrm{NO}_{2}\right)$ of 4-NTP was observed on the functionalized flat gold, whereas the signal was observed on the dendritic fractal. This leads us to conclude that the prepared dendritic fractals are compatible with SEIRA based measurements. To maximize the enhancement for SEIRA, additional design considerations should be considered, notably the presence of an LSPR that is slightly blue-shifted relative to the frequency of vibration for an analyte. Once this has been obtained, further modifications can yield additional enhancement. For example, configuring the structure such that it is on a pedestal has been shown to provide an 
additional order of magnitude of enhancement. ${ }^{27}$ Future studies involving SEIRA on the dendritic fractals should focus on these types of modifications.
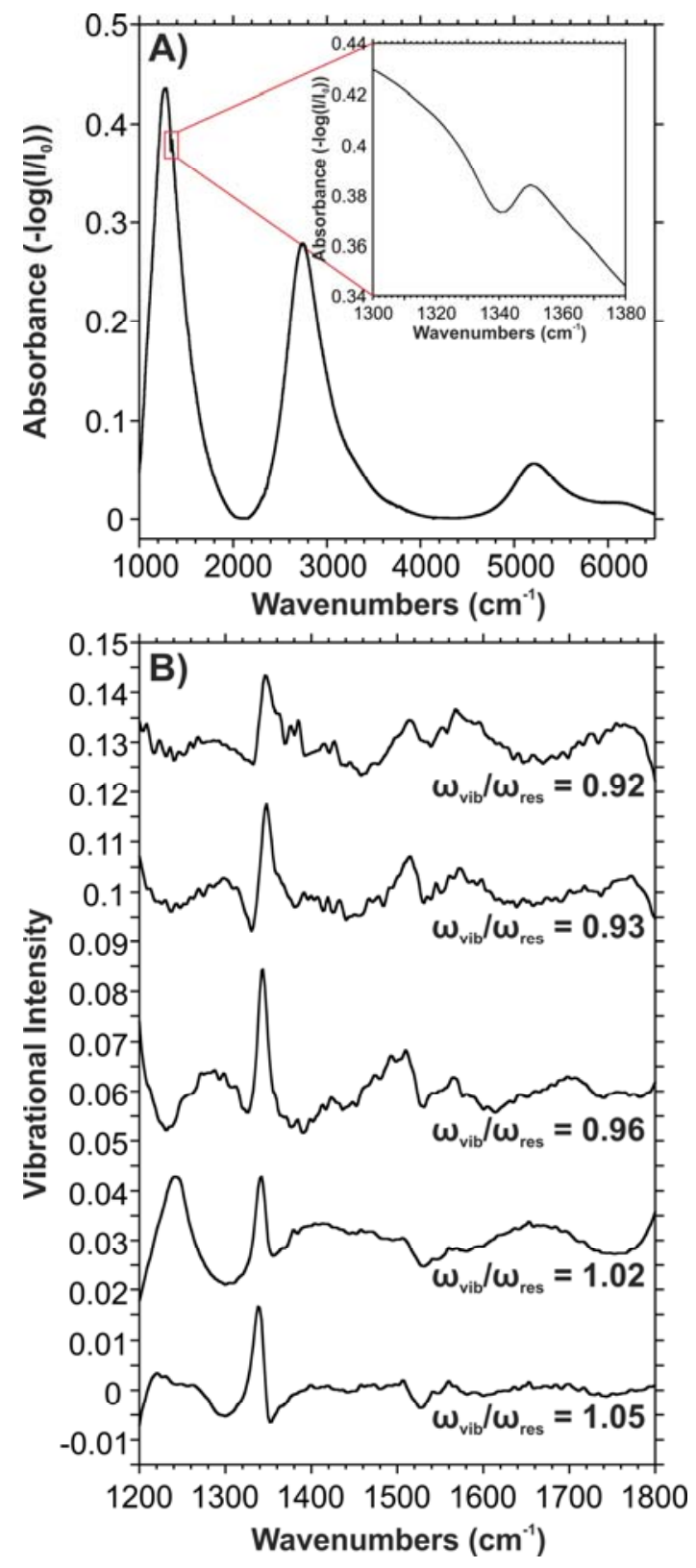

Figure 8. SEIRA measurements of 4-NTP functionalized dendritic fractals. A) Truncated 8 branched second-order generation dendritic fractal (same as Figure 6D). The inset in the figure highlights the symmetric stretch of $\mathrm{NO}_{2}$. B) SEIRA spectra of 4-NTP on different dendritic 
fractals with a polynomial fit having been removed. The spectra are offset for clarity and the ratios of $\omega_{\text {vib }} / \omega_{\text {res }}$ are included.

\section{Conclusion:}

We have demonstrated the design, fabrication, characterization, and application of dendritic fractals for SEIRA. The dendritic structures can generate multiple LSPRs that span the near- and mid-infrared spectral regions. In the first-order generation of the dendritic fractal, there is a single resonance. With each subsequent generation, a new absorption is introduced at lower wavenumbers. These new absorptions correspond to the additional generations, with the lowest energy absorption being the global LSPR of the structure and are tentatively explained using the hybridization model. By tuning the size of the individual nanorods that comprise the dendritic fractal, it is possible to tune spectral position of the absorptions with a high degree of control. Increasing the number of internal branches results in a blue-shift of the higher energy absorptions, and decreases the polarization dependence of the structure. However, as the number of branches in the first-order generation increases, less space exists for the greater number of branches needed in the higher-order generations. Thus, it becomes necessary to truncate the fractal to maintain the rod like structure of the outer branches. These changes do not significantly alter the global LSPR, and instead allow for tuning of the higher energy absorption. We have demonstrated how the lower energy resonances, such as the global LSPR, can be used to detect a molecule of interest when there is spectral overlap between the resonance of the structure and the vibrational mode of the molecule. Overall, the dendritic fractals provide a simple means of preparing nanostructures that exhibit broad optical properties across the near- and mid-infrared spectral ranges. Further work on the dendritic fractal should emphasize optimizing the enhancement of the electromagnetic signal. This can be achieved by altering the configuration of 
the plasmonic platform. Additionally, due to the thin width $(50 \mathrm{~nm})$ of the individual nanorods that make up the dendritic fractal, the structure may also exhibit optical properties in the visible region. These absorptions could then be used for techniques such as SERS and surface-enhanced fluorescence. Such a study could then validate the dendritic fractal as being another structure capable of multispectral molecular plasmonics.

\section{Corresponding Author}

*E-mail address: flagugne@uwo.ca

\section{Conflict of Interest}

The authors declare no competing financial interest.

\section{Acknowledgements}

The authors gratefully acknowledge the Nanofabrication Facility at Western University. This research was supported by the Natural Sciences and Engineering Research Council (NSERC) of Canada. This research was further funded by the Canada Research Chairs program in "Photonics and Nanosciences" (FL-L). G.Q.W thanks NSERC for the postgraduate scholarship doctoral (PGS-D) funding and also acknowledges the receipt of support from the CLS Graduate and PostDoctoral Student Travel Support Program. Research described in this work was performed at the Mid-Infrared Spectromicroscopy (01B1-1) Beamline at the Canadian Light Source, which is supported by the Canada Foundation for Innovation, NSERC, the University of Saskatchewan, the Government of Saskatchewan, Western Economic Diversification, the National Research Council Canada, and the Canadian Institutes of Health Research.

\section{Supplementary Data}


The Supporting Information is available free of charge on the ACS Publications website at DOI:

SEM micrograph showing the reproducibility of the fabrication of the fractal within the $50 \times 50$ $\mu \mathrm{m}^{2}$ patch, SEM micrograph and corresponding absorption spectrum of a patch of fifth-order three branched dendritic fractals, an expanded hybridization model, a comparison of the absorption spectra of the truncated fractals and the traditional fractal one generation prior to truncation, and a comparison of the SEIRA results for the fourth-order three branched dendritic fractal and flat gold.

\section{References}

1. Bhattacharya, R.; Indukuri, C.; Begam, N.; Seeck, O. H.; Basu, J. K., Plasmonic Lipid Bilayer Membranes for Enhanced Detection Sensitivity of Biolabeling Fluorophores. Adv. Funct. Mater. 2015, 25, 7233-7242.

2. Bailey, M. R.; Martin, R. S.; Schultz, Z. D., Role of Surface Adsorption in the SurfaceEnhanced Raman Scattering and Electrochemical Detection of Neurotransmitters. J. Phys. Chem. C 2016, 120, 20624-20633.

3. Henry, A.-I.; Sharma, B.; Cardinal, M. F.; Kurouski, D.; Van Duyne, R. P., SurfaceEnhanced Raman Spectroscopy Biosensing: In Vivo Diagnostics and Multimodal Imaging. Anal. Chem. 2016, 88, 6638-6647.

4. Tabatabaei, M.; Wallace, G. Q.; Caetano, F. A.; Gillies, E. R.; Ferguson, S. S. G.; Lagugne-Labarthet, F., Controlled Positioning of Analytes and Cells on a Plasmonic Platform for Glycan Sensing Using Surface Enhanced Raman Spectroscopy. Chem. Sci. 2016, 7, 575-582. 
5. Wang, W.; Zhang, L.; Li, L.; Tian, Y., A Single Nanoprobe for Ratiometric Imaging and Biosensing of Hypochlorite and Glutathione in Live Cells Using Surface-Enhanced Raman Scattering. Anal. Chem. 2016, 88, 9518-9523.

6. Deckert-Gaudig, T.; Kurouski, D.; Hedegaard, M. A. B.; Singh, P.; Lednev, I. K.; Deckert, V., Spatially Resolved Spectroscopic Differentiation of Hydrophilic and Hydrophobic Domains on Individual Insulin Amyloid Fibrils. Sci. Reports 2016, 6, 33575.

7. Liao, M.; Jiang, S.; Hu, C.; Zhang, R.; Kuang, Y.; Zhu, J.; Zhang, Y.; Dong, Z., TipEnhanced Raman Spectroscopic Imaging of Individual Carbon Nanotubes with Subnanometer Resolution. Nano Lett. 2016, 16, 4040-4046.

8. Choi, H.-K.; Park, W.-H.; Park, C.-G.; Shin, H.-H.; Lee, K. S.; Kim, Z. H., MetalCatalyzed Chemical Reaction of Single Molecules Directly Probed by Vibrational Spectroscopy. J. Am. Chem. Soc. 2016, 138, 4673-4684.

9. dos Santos, D. P.; Temperini, M. L. A.; Brolo, A. G., Single-Molecule Surface-Enhanced (Resonance) Raman Scattering (SE(R)RS) as a Probe for Metal Colloid Aggregation State. J. Phys. Chem. C 2016, 120, 20877-20885.

10. Simoncelli, S.; Roller, E.-M.; Urban, P.; Schreiber, R.; Turberfield, A. J.; Liedl, T.; Lohmüller, T., Quantitative Single-Molecule Surface-Enhanced Raman Scattering by Optothermal Tuning of DNA Origami-Assembled Plasmonic Nanoantennas. ACS Nano 2016, 10, 9809-9815.

11. Ross, M. B.; Mirkin, C. A.; Schatz, G. C., Optical Properties of One-, Two-, and ThreeDimensional Arrays of Plasmonic Nanostructures. J. Phys. Chem. C 2016, 120, 816-830. 
12. Cerjan, B.; Yang, X.; Nordlander, P.; Halas, N. J., Asymmetric Aluminum Antennas for Self-Calibrating Surface-Enhanced Infrared Absorption Spectroscopy. ACS Photonics 2016, 3, $354-360$.

13. Li, Y.; Simeral, M. L.; Natelson, D., Surface-Enhanced Infrared Absorption of SelfAligned Nanogap Structures. J. Phys. Chem. C 2016, 120, 22558-22564.

14. Morhart, T. A.; Quirk, A.; Lardner, M. J.; May, T. E.; Rosendahl, S. M.; Burgess, I. J., Femtomole Infrared Spectroscopy at the Electrified Metal-Solution Interface. Anal. Chem. 2016, 88, 9351-9354.

15. Le Ru, E. C.; Etchegoin, P. G., Rigorous Justification of the $|E| 4$ Enhancement Factor in Surface Enhanced Raman Spectroscopy. Chem. Phys. Lett. 2006, 423, 63-66.

16. Yampolsky, S.; Fishman, D. A.; Dey, S.; Hulkko, E.; Banik, M.; Potma, E. O.; Apkarian, V. A., Seeing a Single Molecule Vibrate Through Time-Resolved Coherent Anti-Stokes Raman Scattering. Nat. Photon. 2014, 8, 650-656.

17. Butet, J.; Brevet, P.-F.; Martin, O. J. F., Optical Second Harmonic Generation in Plasmonic Nanostructures: From Fundamental Principles to Advanced Applications. ACS Nano 2015, 9, 10545-10562.

18. Hou, R.; Shynkar, V.; Lafargue, C.; Kolkowski, R.; Zyss, J.; Lagugne-Labarthet, F., Second Harmonic Generation from Gold Meta-Molecules with Three-Fold Symmetry. Phys. Chem. Chem. Phys. 2016, 18, 7956-7965. 
19. Lagugné-Labarthet, F.; Shen, Y. R., Nonlinear Optical Microscopy. In Optical Imaging and Microscopy: Techniques and Advanced Systems, Török, P.; Kao, F.-J., Eds. Springer Berlin Heidelberg: Berlin, Heidelberg, 2007; pp 237-268.

20. Valev, V. K., Characterization of Nanostructured Plasmonic Surfaces with Second Harmonic Generation. Langmuir 2012, 28, 15454-15471.

21. Kauranen, M.; Zayats, A. V., Nonlinear Plasmonics. Nat. Photon. 2012, 6, 737-748.

22. Rao, G. P. C.; Tharmaraj, V.; Yang, J., Surfactant-Assisted Electroless Deposition of Silver Nanoparticles on Ge Crystal for Ultra-Sensitive Detection by Surface-Enhanced Infrared Absorption Spectroscopy. RSC Adv. 2015, 5, 20390-20395.

23. Rao, G. P. C.; Yang, J., Electroless Reduction of Silver Chloride Precipitates for the Preparation of Highly Sensitive Substrates for Surface-Enhanced Infrared Absorption (SEIRA) Measurements. Appl. Spectrosc. 2015, 69, 37-44.

24. Le, F.; Brandl, D. W.; Urzhumov, Y. A.; Wang, H.; Kundu, J.; Halas, N. J.; Aizpurua, J.; Nordlander, P., Metallic Nanoparticle Arrays: A Common Substrate for Both Surface-Enhanced Raman Scattering and Surface-Enhanced Infrared Absorption. ACS Nano 2008, 2, 707-718.

25. Brown, L. V.; Zhao, K.; King, N.; Sobhani, H.; Nordlander, P.; Halas, N. J., SurfaceEnhanced Infrared Absorption Using Individual Cross Antennas Tailored to Chemical Moieties. J. Am. Chem. Soc. 2013, 135, 3688-3695.

26. Abb, M.; Wang, Y.; Papasimakis, N.; de Groot, C. H.; Muskens, O. L., Surface-Enhanced Infrared Spectroscopy Using Metal Oxide Plasmonic Antenna Arrays. Nano Lett. 2014, 14, 346352. 
27. Huck, C.; Toma, A.; Neubrech, F.; Chirumamilla, M.; Vogt, J.; De Angelis, F.; Pucci, A., Gold Nanoantennas on a Pedestal for Plasmonic Enhancement in the Infrared. ACS Photonics 2015, 2, 497-505.

28. Limaj, O.; Etezadi, D.; Wittenberg, N. J.; Rodrigo, D.; Yoo, D.; Oh, S.-H.; Altug, H., Infrared Plasmonic Biosensor for Real-Time and Label-Free Monitoring of Lipid Membranes. Nano Lett. 2016, 16, 1502-1508.

29. Aksu, S.; Yanik, A. A.; Adato, R.; Artar, A.; Huang, M.; Altug, H., High-Throughput Nanofabrication of Infrared Plasmonic Nanoantenna Arrays for Vibrational Nanospectroscopy. Nano Lett. 2010, 10, 2511-2518.

30. Aksu, S.; Cetin, A. E.; Adato, R.; Altug, H., Plasmonically Enhanced Vibrational Biospectroscopy Using Low-Cost Infrared Antenna Arrays by Nanostencil Lithography. Adv. Opt. Mater. 2013, 1, 798-803.

31. Zhang, M.; Bechstein, D. J. B.; Wilson, R. J.; Wang, S. X., Wafer-Scale Synthesis of Monodisperse Synthetic Magnetic Multilayer Nanorods. Nano Lett. 2014, 14, 333-338.

32. Bagheri, S.; Weber, K.; Gissibl, T.; Weiss, T.; Neubrech, F.; Giessen, H., Fabrication of Square-Centimeter Plasmonic Nanoantenna Arrays by Femtosecond Direct Laser Writing Lithography: Effects of Collective Excitations on SEIRA Enhancement. ACS Photonics 2015, 2, $779-786$.

33. Braun, A.; Maier, S. A., Versatile Direct Laser Writing Lithography Technique for Surface Enhanced Infrared Spectroscopy Sensors. ACS Sens. 2016, 1, 1155-1162. 
34. Cetin, A. E.; Korkmaz, S.; Durmaz, H.; Aslan, E.; Kaya, S.; Paiella, R.; Turkmen, M., Quantification of Multiple Molecular Fingerprints by Dual-Resonant Perfect Absorber. Adv. Opt. Mater. 2016, 4, 1274-1280.

35. Wallace, G. Q.; Tabatabaei, M.; Hou, R.; Coady, M. J.; Norton, P. R.; Simpson, T. S.; Rosendahl, S. M.; Merlen, A.; Lagugné-Labarthet, F., Superimposed Arrays of Nanoprisms for Multispectral Molecular Plasmonics. ACS Photonics 2016, 3, 1723-1732.

36. Chen, K.; Adato, R.; Altug, H., Dual-Band Perfect Absorber for Multispectral PlasmonEnhanced Infrared Spectroscopy. ACS Nano 2012, 6, 7998-8006.

37. Hartstein, A.; Kirtley, J. R.; Tsang, J. C., Enhancement of the Infrared Absorption from Molecular Monolayers with Thin Metal Overlayers. Phys. Rev. Lett. 1980, 45, 201-204.

38. Aroca, R. F.; Ross, D. J.; Domingo, C., Surface-Enhanced Infrared Spectroscopy. Appl. Spectrosc. 2004, 58, 324A-338A.

39. Neubrech, F.; Huck, C.; Weber, K.; Pucci, A.; Giessen, H., Surface-Enhanced Infrared Spectroscopy Using Resonant Nanoantennas. Chem. Rev. 2017, DOI: 10.1021/acs.chemrev.6b00743.

40. Neuman, T.; Huck, C.; Vogt, J.; Neubrech, F.; Hillenbrand, R.; Aizpurua, J.; Pucci, A., Importance of Plasmonic Scattering for an Optimal Enhancement of Vibrational Absorption in SEIRA with Linear Metallic Antennas. J. Phys. Chem. C 2015, 119, 26652-26652.

41. De Zuani, S.; Reindl, T.; Rommel, M.; Gompf, B.; Berrier, A.; Dressel, M., High-Order Hilbert Curves: Fractal Structures with Isotropic, Tailorable Optical Properties. ACS Photonics 2015, 2, 1719-1724. 
42. Gottheim, S.; Zhang, H.; Govorov, A. O.; Halas, N. J., Fractal Nanoparticle Plasmonics: The Cayley Tree. ACS Nano 2015, 9, 3284-3292.

43. Aslan, E.; Aslan, E.; Wang, R.; Hong, M. K.; Erramilli, S.; Turkmen, M.; Saracoglu, O. G.; Dal Negro, L., Multispectral Cesaro-Type Fractal Plasmonic Nanoantennas. ACS Photonics 2016, 3, 2102-2111.

44. Hasan, D.; Ho, C. P.; Lee, C., Realization of Fractal-Inspired Thermoresponsive Quasi3D Plasmonic Metasurfaces with EOT-Like Transmission for Volumetric and Multispectral Detection in the Mid-IR Region. ACS Omega 2016, 1, 818-831.

45. Volpe, G.; Volpe, G.; Quidant, R., Fractal Plasmonics: Subdiffraction Focusing and Broadband Spectral Response by a Sierpinski Nanocarpet. Opt. Express 2011, 19, 3612-3618.

46. Hsu, K. H.; Back, J. H.; Fung, K.-H.; Ferreira, P. M.; Shim, M.; Fang, N. X., SERS EM Field Enhancement Study Through Fast Raman Mapping of Sierpinski Carpet Arrays. J. Raman Spectrosc. 2010, 41, 1124-1130.

47. Guanhai, L.; Xiaoshuang, C.; Bo, N.; Oupeng, L.; Lujun, H.; Yuan, J.; Weida, H.; Wei, L., Fractal H-Shaped Plasmonic Nanocavity. Nanotechnol. 2013, 24, 205702.

48. Hegde, R. S.; Khoo, E. H., Broadband Optical Response in Ternary Tree Fractal Plasmonic Nanoantenna. Plasmonics 2016, 11, 465-473.

49. Kischkat, J.; Peters, S.; Gruska, B.; Semtsiv, M.; Chashnikova, M.; Klinkmüller, M.; Fedosenko, O.; Machulik, S.; Aleksandrova, A.; Monastyrskyi, G. et al., Mid-Infrared Optical Properties of Thin Films of Aluminum Oxide, Titanium Dioxide, Silicon Dioxide, Aluminum Nitride, and Silicon Nitride. Appl. Opt. 2012, 51, 6789-6798. 
50. Lehmann, F.; Richter, G.; Borzenko, T.; Hock, V.; Schmidt, G.; Molenkamp, L. W., Fabrication of Sub-10-nm Au-Pd Structures Using $30 \mathrm{keV}$ Electron Beam Lithography and Liftoff. Microelectron. Eng. 2003, 65, 327-333.

51. Palik, E. D., In Handbook of Optical Constants of Solids, Academic Press: Burlington, 1997.

52. Shih, W.-C.; Santos, G. M.; Zhao, F.; Zenasni, O.; Arnob, M. M. P., Simultaneous Chemical and Refractive Index Sensing in the 1-2.5 $\mu \mathrm{m}$ Near-Infrared Wavelength Range on Nanoporous Gold Disks. Nano Lett. 2016, 16, 4641-4647.

53. Kearns, H.; Bedics, M. A.; Shand, N. C.; Faulds, K.; Detty, M. R.; Graham, D., Sensitive SERS Nanotags for use with 1550 nm (Retina-Safe) Laser Excitation. Analyst 2016, 141, 50625065.

54. Prodan, E.; Radloff, C.; Halas, N. J.; Nordlander, P., A Hybridization Model for the Plasmon Response of Complex Nanostructures. Science 2003, 302, 419-422.

55. Larsson, E. M.; Hao, F.; Eurenius, L.; Olsson, E.; Nordlander, P.; Sutherland, D. S., Plasmon Hybridization in Stacked Double Gold Nanorings with Reduced Symmetry. Small 2008, 4, 1630-1634.

56. Fang, Z.; Thongrattanasiri, S.; Schlather, A.; Liu, Z.; Ma, L.; Wang, Y.; Ajayan, P. M.; Nordlander, P.; Halas, N. J.; Garcia de Abajo, F. J., Gated Tunability and Hybridization of Localized Plasmons in Nanostructured Graphene. ACS Nano 2013, 7, 2388-2395. 
57. Vedraine, S.; Hou, R.; Norton, P. R.; Lagugné-Labarthet, F., On the Absorption and Electromagnetic Field Spectral Shifts in Plasmonic Nanotriangle Arrays. Opt. Express 2014, 22, 13308-13313.

58. Shimada, T.; Nakashima, H.; Kumagai, Y.; Ishigo, Y.; Tsushima, M.; Ikari, A.; Suzuki, Y., What is the Key Structural Parameter for Infrared Absorption Enhancement on Nanostructures? J. Phys. Chem. C 2016, 120, 534-541.

59. D’Andrea, C.; Bochterle, J.; Toma, A.; Huck, C.; Neubrech, F.; Messina, E.; Fazio, B.; Maragò, O. M.; Di Fabrizio, E.; Lamy de La Chapelle, M. et al., Optical Nanoantennas for Multiband Surface-Enhanced Infrared and Raman Spectroscopy. ACS Nano 2013, 7, 3522-3531.

60. Merklin, G. T.; He, L.-T.; Griffiths, P. R., Surface-Enhanced Infrared Absorption Spectrometry of p-Nitrothiophenol and its Disulfide. Appl. Spectrosc. 1999, 53, 1448-1453.

61. Verger, F.; Nazabal, V.; Colas, F.; Němec, P.; Cardinaud, C.; Baudet, E.; Chahal, R.; Rinnert, E.; Boukerma, K.; Peron, I. et al., RF Sputtered Amorphous Chalcogenide Thin Films for Surface Enhanced Infrared Absorption Spectroscopy. Opt. Mater. Express 2013, 3, 21122131.

62. Neubrech, F.; Pucci, A.; Cornelius, T. W.; Karim, S.; Garcia-Etxarri, A.; Aizpurua, J., Resonant Plasmonic and Vibrational Coupling in a Tailored Nanoantenna for Infrared Detection. Phys. Rev. Lett. 2008, 101, 157403.

63. Vogt, J.; Huck, C.; Neubrech, F.; Toma, A.; Gerbert, D.; Pucci, A., Impact of the Plasmonic Near- and Far-Field Resonance-Energy Shift on the Enhancement of Infrared Vibrational Signals. Phys. Chem. Chem. Phys. 2015, 17, 21169-21175. 


\section{TOC GRAPHIC}

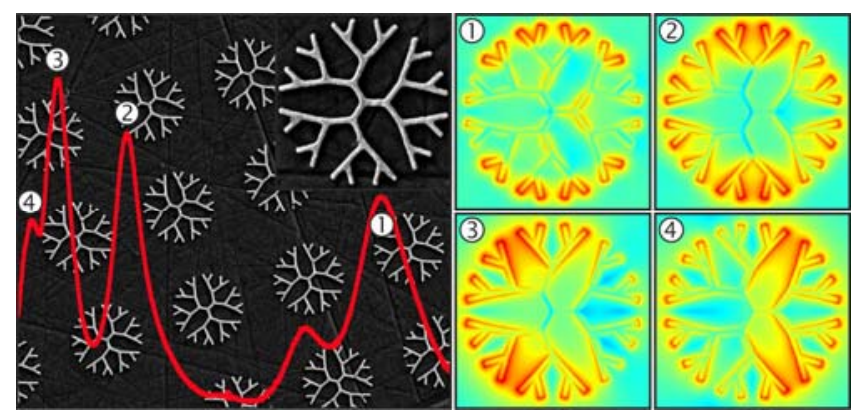

